\title{
Structural and Functional Brain Alterations in Post-traumatic Headache Attributed to Mild Traumatic Brain Injury: A Narrative Review
}

\author{
Todd J. Schwedt* \\ Department of Neurology, Mayo Clinic, Scottsdale, AZ, United States
}

Introduction: By definition, post-traumatic headache $(\mathrm{PTH})$ attributed to mild traumatic brain injury (mTBI) is not associated with brain structural abnormalities that are seen on routine clinical inspection of brain images. However, subtle brain structural abnormalities, as well as functional abnormalities, detected via research imaging techniques yield insights into the pathophysiology of PTH. The objective of this manuscript is to summarize published findings regarding research imaging of the brain in PTH attributed to mTBI.

OPEN ACCESS

Edited by:

Massimo Filippi,

Vita-Salute San Raffaele

University, Italy

Reviewed by:

Gianluca Coppola,

Fondazione G. B. Bietti (IRCCS), Italy

Marco Carotenuto,

Università degli Studi della Campania

Luigi Vanvitelli Caserta, Italy

*Correspondence:

Todd J. Schwedt

schwedt.todd@mayo.edu

Specialty section:

This article was submitted to

Headache Medicine and Facial Pain,

a section of the journal

Frontiers in Neurology

Received: 01 April 2019

Accepted: 24 May 2019

Published: 14 June 2019

Citation:

Schwedt TJ (2019) Structural and

Functional Brain Alterations in

Post-traumatic Headache Attributed

to Mild Traumatic Brain Injury: A Narrative Review.

Front. Neurol. 10:615.

doi: 10.3389/fneur.2019.00615
Methods: For this narrative review, PubMed was searched using the terms "post-traumatic headache" or "post-concussion headache" and "imaging" or "magnetic resonance imaging" or "research imaging" or "positron emission tomography". Articles were chosen for inclusion based on their relevance to the topic.

Results: Ten articles were ultimately included within this review. The studies investigated white matter tract integrity and functional connectivity in acute PTH, structural measures, white matter tract integrity, cerebral blood flow, and functional connectivity in persistent PTH (PPTH), and proton spectroscopy in both acute and persistent PTH. The articles demonstrate that acute and persistent PTH are associated with abnormalities in brain structure, that acute and persistent PTH are also associated with abnormalities in brain function, that it might be possible to predict the persistence of PTH using brain imaging findings, and that there are differences in imaging findings when comparing PTH to healthy controls and when comparing PTH to migraine. Although it is not entirely clear if the imaging findings are directly attributable to PTH as opposed to the underlying TBI or other post-TBI symptoms, correlations between the imaging findings with headache frequency and headache resolution suggest a true relationship between the imaging findings and PTH.

Conclusions: PTH attributed to mTBI is associated with abnormalities in brain structure and function that can be detected via research imaging. Additional studies are needed to determine the specificity of the findings for $\mathrm{PTH}$, to differentiate findings attributed to PTH from those attributed to the underlying TBI and coexistent post-TBI symptoms, and to determine the accuracy of imaging findings for predicting the development of PPTH.

Keywords: post-traumatic headache, traumatic brain injury, concussion, brain imaging, migraine, functional magnetic resonance imaging, diffusion tensor imaging, functional connectivity 


\section{INTRODUCTION}

Post-traumatic headache $(\mathrm{PTH})$ attributed to traumatic injury to the head is defined by the International Classification of Headache Disorders 3 (ICHD-3) as a headache that is reported to have developed within 7 days of the head injury, regaining consciousness following the head injury, or discontinuation of medication(s) impairing the ability to sense or report headache following the head injury (1). PTH can be a new headache that develops after the injury or it can be significant worsening of a pre-existing headache, such as when there is substantial worsening of migraine patterns following the injury. PTH is considered "acute" when it has been present for 3 months or less, and "persistent" when it has been present for more than 3 months. According to the ICHD-3, PTH can be attributed to mild traumatic head (brain) injury or to moderate or severe traumatic head (brain) injury. An essential component of the ICHD-3 definition for mild traumatic brain injury (mTBI) is that there is no imaging evidence of a traumatic head injury such as skull fracture, intracranial hemorrhage, and/or brain contusion. Thus, by this definition, brain images from individuals with mTBI are clinically interpreted as "normal." However, as discussed within this article, advanced imaging techniques and imaging data analyses reveal abnormalities in brain structure and function in individuals with PTH attributed to mTBI.

PTH is a highly prevalent symptom following TBI; likely, it is the most common acute and persistent symptom following mTBI (2). Although PTH may be the only symptom during the acute and persistent phases following TBI, often PTH is accompanied by cognitive, mood, sleep, and autonomic symptoms $(3,4)$. The phenotype of PTH most commonly resembles that of migraine, due to the presence of moderate to severe headache and sensory hypersensitivities, or tension-type headache, although less commonly it resembles other primary headaches $(5,6)$. Given the phenotypic overlap with primary headaches, like migraine, it is presumed that there are shared underlying mechanisms between PTH and the primary headaches.

Given the high incidence of PTH following TBI, the significant impact that PTH has on individuals, and the absence of PTH-specific therapies, it is essential that we continue to investigate mechanisms for acute PTH and PTH persistence. Research neuroimaging of brain structure and function is one useful method by which to conduct such investigations. In this manuscript, published findings from research neuroimaging studies of acute and persistent PTH are summarized and interpreted, and future directions are discussed.

\section{METHODS}

For this narrative review, articles were identified by searching PubMed using the terms "post-traumatic headache" or "post-concussion headache" and "imaging" or "magnetic resonance imaging" or "research imaging" or "positron emission tomography." The resulting list was reviewed by the author and abstracts and full manuscripts were further reviewed based on the author's judgment of the relevance of each article to the topic. The reference lists of each selected article were reviewed to identify additional publications not identified via the PubMed search. Ultimately, 10 articles were included in this Review.

\section{IMAGING ACUTE PTH (TABLE 1)}

Diffusion tensor imaging (DTI) allows for assessment of white matter integrity. Alhilali and colleagues performed a DTI analysis of 58 individuals who had PTH of a migraine phenotype attributed to mTBI vs. 17 individuals who had mTBI without PTH of a migraine phenotype (although they could have PTH of a non-migraine phenotype) (7). The median time from injury to evaluation was 20 days. The cohort with PTH of a migraine phenotype had lower fractional anisotropy (FA) in the corpus callosum and fornix/septohippocampal circuit. Furthermore, FA within regions of the fornix/septohippocampal circuit correlated positively with visual memory performance $(r=0.325, p=$ 0.01). The corpus callosum and the fornix/septohippocampal circuit have previously been implicated in migraine or cortical spreading depression pathophysiology $(10,11)$. A major strength of this study is the use of a mTBI group without PTH of a migraine phenotype as a comparator group, theoretically controlling for potential effects of the mTBI on white matter tract integrity. However, it is not clear what proportion of this control group had PTH of a phenotype other than migraine and how the presence of such headaches could impact DTI findings. Furthermore, the correlation between DTI measures in the fornix/septohippocampal circuit with visual memory performance could suggest that the DTI finding is not (entirely) related to the presence of $\mathrm{PTH}$ of a migraine phenotype.

A principal components analysis of DTI FA data was performed to identify white matter injury patterns associated with PTH attributed to mTBI (9). Sixty-four patients with mTBI were included, 40 with PTH and 24 without PTH. Median duration between injury and presentation was between 3 and 4 weeks. A principal component that included decreased FA in the splenium and increased FA in the genu of the corpus callosum was associated with an increased risk of PTH (odds ratio 2.32, 95\% confidence interval 1.29-4.67, $p=0.01$ ). This principal component accurately classified those who had PTH with an area under the curve of 0.73 .

Delic and colleagues investigated the performance of a classifier based on Shannon entropy (SE) that used FA DTI data in differentiating individuals who had PTH of a migraine phenotype attributed to mTBI from those who had mTBI without PTH of a migraine phenotype and from healthy controls (8). SE measures the complexity of a dataset-the more information or complexity within a dataset, the higher the SE. In the realm of white matter integrity, higher SE of FA data could be reflective of a mixed pattern of white matter tract injury, swelling, and remyelination. It has been hypothesized that SE provides a more accurate measurement of axonal changes that occur after neurologic injury. For this analysis, 57 participants with mTBI and PTH of a migraine phenotype, 17 with mTBI without PTH 
TABLE 1 | Imaging findings during acute post-traumatic headache.

\begin{tabular}{|c|c|c|c|c|}
\hline References & PTH phase & $\begin{array}{l}\text { Imaging modality or } \\
\text { sequence }\end{array}$ & Comparison group(s) & Main findings \\
\hline Alhilali et al. (7) & Acute & DTI & $\begin{array}{l}\text { mTBI with PTH of migraine phenotype } \\
\text { vs. mTBI without PTH of migraine phenotype }\end{array}$ & $\begin{array}{l}\text { PTH of migraine phenotype: lower FA in corpus callosum } \\
\text { and fornix/septohippocampal circuit. }\end{array}$ \\
\hline Delic et al. (8) & Acute & DTI & $\begin{array}{l}\text { 1) mTBI with PTH of migraine phenotype vs. } \\
\text { mTBI without PTH of migraine phenotype } \\
\text { 2) mTBI with PTH of migraine phenotype vs. } \\
\text { migraine controls and vs. HC }\end{array}$ & $\begin{array}{l}\text { mTBI with PTH of migraine phenotype vs. all other groups: } \\
\text { lower SE of FA data } \\
\text { Inverse correlation between SE and time to recovery. } \\
\text { Accuracy for differentiating PTH of migraine phenotype from } \\
\text { a) controls: specificity } 95 \% \text {, sensitivity } 77 \% \\
\text { b) } \mathrm{mTBI} \text { without PTH of a migraine phenotype: specificity } \\
75 \% \text {, sensitivity } 81 \%\end{array}$ \\
\hline
\end{tabular}

Ghodadra et al. (9) Acute DTI mTBI with PTH vs. mTBI without PTH

PTH: lower FA in splenium of corpus callosum; higher FA in genu of corpus callosum

Diffusion tensor imaging studies demonstrate anomalies in white matter tract integrity in acute PTH due to mild traumatic brain injury.

DTI, diffusion tensor imaging; mTBI, mild traumatic brain injury; PTH, post-traumatic headache; FA, fractional anisotropy; HC, healthy control subject; SE, Shannon Entropy.

TABLE 2 | Imaging studies that investigated acute and persistent post-traumatic headache or prediction of persistent post-traumatic headache.

\begin{tabular}{|c|c|c|c|c|}
\hline References & PTH phase & $\begin{array}{l}\text { Imaging modality or } \\
\text { sequence }\end{array}$ & Comparison group(s) & Main findings \\
\hline Obermann et al. (12)* & $\begin{array}{l}\text { 1) Acute } \\
\text { 2) Persistent }\end{array}$ & Gray matter density & $\begin{array}{l}\text { 1) Acute PTH vs. HC } \\
\text { 2) PTH-acute vs. persistent }\end{array}$ & $\begin{array}{l}\text { Acute PTH vs. HC: no differences } \\
\text { PPTH vs. HC: less gray matter density in anterior cingulate } \\
\text { cortex and dorsolateral prefrontal cortex in PPTH } \\
12 \text { months: those who had PPTH at } 3 \text { months, but resolved } \\
\text { prior to } 12 \text { months had normal gray matter density }\end{array}$ \\
\hline Niu et al. (14) & $\begin{array}{l}\text { Acute: predicting } \\
\text { development of PPTH }\end{array}$ & Functional connectivity & $\begin{array}{l}\text { PPTH vs. PTH resolution } \\
\text { during acute phase }\end{array}$ & $\begin{array}{l}\text { Connectivity of PAG with inferior parietal lobule and with } \\
\text { precuneus predicted persistence of PTH: } 100 \% \text { sensitivity and } \\
78 \% \text { specificity }\end{array}$ \\
\hline
\end{tabular}

A study of gray matter density in those with PTH attributed to whiplash showed less gray matter in PPTH, but not acute PTH, compared with healthy controls. An MRS study that combined individuals with acute PTH with those who had PPTH into one group, found evidence for reduced neuronal vitality, increased membrane turnover and cell proliferation in PTH. A functional connectivity study demonstrated promise for using measures of periaqueductal gray connectivity for predicting the persistence of PTH.

PTH, post-traumatic headache; HC, healthy control subject; MRS, magnetic resonance spectroscopy; NAA, N-acetylspartate; PPTH, persistent post-traumatic headache; PAG, periaqueductal gray. ${ }^{*}$ PTH attributed to whiplash.

of a migraine phenotype, 22 healthy control subjects, and 20 control subjects with migraine were included. All mTBI patients had acute PTH with a median time from injury to clinical presentation of 20 days and with $83 \%$ of patients presenting within 2 months of injury. Those with mTBI were found to have lower SE compared to controls and there was an inverse correlation between SE and time to patient recovery. PTH of a migraine phenotype was associated with lower SE compared to mTBI without PTH of a migraine phenotype. SE measures provided high accuracy for differentiating those with mTBI from controls (Area under the curve $=0.92$, specificity $95 \%$, sensitivity $77 \%$ ) and for differentiating mTBI with $\mathrm{PTH}$ of a migraine phenotype from mTBI without PTH of a migraine phenotype (Area under the curve $=0.85$, specificity $75 \%$, sensitivity $81 \%$ ). This study suggests that SE analyses of DTI data may be particularly useful for investigating white matter pathology and repair following mTBI and for building classification models (i.e., diagnostic biomarkers) for PTH.

\section{COMBINED STUDY OF ACUTE AND PERSISTENT PTH USING MR SPECTROSCOPY (TABLE 2)}

MRI proton spectroscopy (MRS) was used to compare Nacetylspartate (NAA, an indicator of neuronal vitality) and choline (a marker of membrane turnover) ratios with creatinine in 17 individuals with PTH attributed to mTBI vs. 12 healthy controls (13). Nine individuals had acute PTH while the remaining 8 had PPTH. Compared to controls, PTH was associated with reduced NAA/creatinine in the anterior regions of the frontal lobe white matter, anterior and posterior medial 
regions of the frontal lobes, and medial regions of the parietal lobes. Choline/creatinine was increased in regions of the posterior frontal lobe white matter, anterior medial frontal lobe, and medial parietal lobes. Authors concluded that decreased NAA is suggestive of reduced neuronal vitality, likely due to the underlying head trauma, while the increased choline is a marker of membrane turnover and cell proliferation developing around the injured neurons.

\section{PREDICTING THE PERSISTENCE OF PTH USING BRAIN IMAGING}

Fortunately, many individuals with acute PTH have headache resolution during the first few days to weeks following PTH onset and thus do not have persistence of PTH (2). However, up to $60 \%$ do not have $\mathrm{PTH}$ resolution, their $\mathrm{PTH}$ endures for more than 3 months, and they are classified as having PPTH (2). The ability to predict who will have persistence of PTH could be valuable for several reasons: (1) those at high risk for PPTH might benefit from closer clinical follow-up; (2) early intervention might reduce the impact of PTH and might prevent PTH persistence (although specific interventions for preventing PTH persistence have yet to be identified); and (3) those individuals at high risk for PPTH would be appropriate for clinical trials of therapeutics aimed at treating acute PTH and reducing the risk for PTH persistence.

An MRI resting-state functional connectivity study by Niu and colleagues suggests that prediction of PPTH is possible (14). In this study, 54 patients with mTBI and acute PTH had brain MRIs including functional connectivity measurements within 7 days of their injury, and they had a 3-month follow-up evaluation to assess for PTH persistence. Investigators analyzed functional connectivity with the right ventrolateral periaqueductal gray (PAG), since this region has been shown to have abnormal connectivity in other pain studies, since it is vulnerable to mTBI, and since it plays a role in opioid antinociception (15-17). At baseline, compared to healthy controls, those with acute PTH had weaker right ventrolateral PAG functional connectivity with several regions of the default mode network, including regions in the right and left precuneus, right inferior parietal lobule, and right angular gyrus, and stronger connectivity with a region in the left middle temporal gyrus. There were significant negative correlations between PAG with right precuneus connectivity and Headache Impact Test 6 scores and between PAG with right inferior parietal lobule and Headache Impact Test 6 scores when measured at baseline. Furthermore, baseline connectivity between the PAG with the right inferior parietal lobule and with the right precuneus were predictors for having persistence of PTH measured at 3 months. A logistic regression model including age, sex, years of education, loss of consciousness duration, and functional connectivity of the PAG with the default mode network predicted PPTH with 100\% sensitivity $(95 \%$ confidence interval (CI) 66\%-100\%) and 78\% specificity (95\% CI $63 \%-89 \%)$. This study demonstrates that acute PTH is associated with altered functional connectivity of the PAG with regions of the default mode network, that the connectivity strength correlates with PTH burden, and that functional connectivity measured during the acute post-TBI phase contributes to prediction of PTH persistence.

\section{IMAGING PERSISTENT PTH (TABLE 3)}

An MRI study by Chong and colleagues compared vertexby-vertex cortical thickness measurements in 33 patients with PPTH due to mTBI with 33 healthy controls (18). Those with PPTH had a median of 7 years with PPTH and 16 days per month with headache and had no history of migraine prior to TBI. After adjustment for several factors that could impact cortical thickness, including age, sex, depression scores, and anxiety scores, the PPTH group had significantly less cortical thickness in left and right frontal (superior frontal, caudal middle frontal, precentral) and right parietal (precuneus, supramarginal, inferior parietal, superior parietal) regions compared to healthy controls. Considering these regions that differed between subject groups, there were significant negative correlations between the thickness of the left and right superior frontal regions with headache frequency. There were no significant correlations between the cortical thickness of these regions and years lived with PPTH. These findings suggest a relationship between the severity of PTH (measured as headache frequency in this case) with brain structure. Relationships between the severity of postTBI symptoms with measures of brain structure have been suggested in other studies, although not specifically investigating PTH $(23,24)$. The association between headache frequency with cortical thickness, but not years of PTH with cortical thickness, at least suggests that the finding of less cortical thickness is attributable to PTH as opposed to the underlying mTBI.

Studies that compare brain imaging findings in those with PPTH to individuals with other headache types, like migraine, can provide insights into shared and distinct pathophysiology. Regional cerebral blood flow, measured by the xenon-133 inhalation technique, was investigated in 35 individuals with PPTH attributed to mTBI vs. 49 non-headache controls and 92 individuals with migraine (20). Compared to healthy controls and individuals with migraine, those with PPTH had reduced regional cerebral blood flow, and greater regional and hemispheric asymmetries. The authors suggested that cerebral blood flow changes and vasomotor instability may be contributing mechanisms to the development of PTH and other post-TBI symptoms.

An MRI investigation of regional volumes, cortical thickness, surface area, and brain curvature found structural differences between PPTH and migraine cohorts in the right lateral orbitofrontal lobe, left caudal middle frontal lobe, left superior frontal lobe, left precuneus, and right supramarginal gyrus (21). Amongst these regions that differed between PPTH and migraine, the structure of regions in the right lateral orbitofrontal lobe, right supramarginal gyrus, and left superior frontal lobe also differed between PPTH and healthy controls who had no history of head trauma or migraine. A DTI study by Chong and colleagues investigated node-by-node white matter tract integrity in PPTH vs. migraine and vs. healthy controls (19). 
TABLE 3 | Imaging findings during persistent post-traumatic headache.

\begin{tabular}{lllll}
\hline References & PTH phase & $\begin{array}{l}\text { Imaging modality or } \\
\text { measure }\end{array}$ & $\begin{array}{l}\text { Comparison } \\
\text { group(s) }\end{array}$ & Main findings \\
\hline $\begin{array}{l}\text { Chong et al. } \\
\text { (18) }\end{array}$ & Persistent & Cortical thickness & HC & $\begin{array}{l}\text { Less thickness in frontal (superior, caudal middle, precentral) and parietal lobes } \\
\text { (precuneus, supramarginal, inferior, superior). }\end{array}$ \\
& & & Negative correlations between superior frontal thickness with headache frequency.
\end{tabular}

\begin{tabular}{lll}
\hline Chong et al. Persistent & DTI & 1) Migraine \\
(19) & & 2) $\mathrm{HC}$
\end{tabular}

vs. Migraine: DTI differences in anterior thalamic radiations, cingulum, inferior and

superior longitudinal fasciculi, uncinate fasciculi, corticospinal tract.

Positive correlation between cingulum angular bundle MD and RD with headache frequency.

vs. HC: DTI differences in anterior thalamic radiations, cingulum, corticospinal tract, inferior longitudinal fasciculus, uncinate fasciculus, forceps major and minor.

\begin{tabular}{|c|c|c|c|c|}
\hline $\begin{array}{l}\text { Gilkey et al. } \\
(20)\end{array}$ & Persistent & $\mathrm{CBF}$ & $\begin{array}{l}\text { 1) Migraine } \\
\text { 2) } \mathrm{HC}\end{array}$ & $\begin{array}{l}\text { vs. Migraine + HC: reduced regional CBF; greater regional and hemispheric CBF } \\
\text { asymmetries. }\end{array}$ \\
\hline $\begin{array}{l}\text { Schwedt } \\
\text { et al. }(21)\end{array}$ & Persistent & $\begin{array}{l}\text { Regional volumes, } \\
\text { cortical thickness, } \\
\text { surface area, curvature }\end{array}$ & $\begin{array}{l}\text { 1) Migraine } \\
\text { 2) } \mathrm{HC}\end{array}$ & $\begin{array}{l}\text { vs. Migraine: structural differences in frontal (lateral orbitofrontal, caudal middle, } \\
\text { superior) and parietal lobes (precuneus, supramarginal). } \\
\text { vs. HC (only considering regions that differed between PPTH vs. Migraine): frontal } \\
\text { (lateral orbitofrontal, superior) and parietal lobes (supramarginal). }\end{array}$ \\
\hline
\end{tabular}

\begin{tabular}{llll}
\hline Dumkrieger & Persistent & Static and dynamic & 1) Migraine \\
et al. (22) & & functional connectivity & 2) HC
\end{tabular}

\begin{abstract}
vs. Migraine (static connectivity): 17 region pairs that included somatosensory, insula, hypothalamus, anterior and middle cingulate, temporal pole, supramarginal, superior parietal, middle occipital, lingual, pulvinar, precuneus, cuneus, somatomotor, ventromedial prefrontal, and dorsolateral prefrontal regions.

Correlations between years with headache and headache frequency with static connectivity of a few region pairs.

vs. Migraine (dynamic connectivity): 10 region pairs that included somatosensory, hypothalamus, middle cingulate, temporal pole, supramarginal, superior parietal, lingual gyrus, somatomotor, precentral, posterior cingulate, middle frontal, fusiform, parieto-occipital, and amygdala regions.

Correlations between headache frequency and pain intensity with dynamic connectivity with a couple region pairs.

vs. HC (only considering regions that differed between PPTH vs. Migraine; static connectivity): dorsolateral prefrontal, ventromedial prefrontal, middle cingulate, somatomotor, pulvinar, insula, hypothalamus.

vs. HC (only considering regions that differed between PPTH vs. Migraine; dynamic connectivity): somatosensory, lingual, middle cingulate, supramarginal, temporal pole, middle frontal.
\end{abstract}

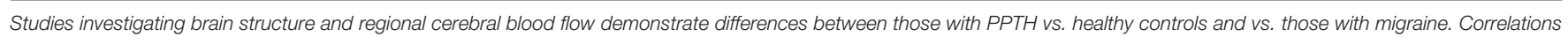
between some of the structural measures with headache frequency provide additional evidence for a relationship between the structural changes with PPTH.

$H C$, healthy control subject; DTI, diffusion tensor imaging; $M D$, mean diffusivity; RD, radial diffusivity; $C B F$, cerebral blood flow; PPTH, persistent post-traumatic headache.

DTI measures (mean diffusivity or radial diffusivity) differed in PPTH vs. migraine for several white matter tracts including the anterior thalamic radiations, cingulum, inferior longitudinal fasciculi, uncinate fasciculi, corticospinal tract, and superior longitudinal fasciculi. Amongst those with PPTH there was a positive correlation between cingulum angular bundle mean diffusivity and radial diffusivity with headache frequency, a relationship not seen amongst those with migraine. A study by Dumkrieger and colleagues compared static and dynamic functional connectivity of 59 regions of interest involved in pain processing in PPTH vs. migraine (22). Between group differences in static and dynamic functional connectivity were identified for several regions and correlations between connectivity strength and headache burden (i.e., years with headache, headache frequency) were found. These studies demonstrate that despite significant overlap in symptoms between migraine and PPTH
(89-96\% of those with PPTH in these studies had a phenotype consistent with "migraine" or "probable migraine"), there are identifiable differences in brain structure and function, perhaps suggesting distinct underlying pathophysiology. Further studies are necessary to determine the specificity of the differences for PPTH and to determine if the findings are directly related to PPTH vs. being attributable to the underlying $\mathrm{mTBI}$ irrespective of PPTH.

\section{LIMITATIONS AND FUTURE DIRECTIONS}

One of the biggest challenges in research imaging of PTH is differentiating imaging findings associated with PTH vs. those attributable to the underlying TBI vs. those associated with other post-TBI symptoms. mTBI is associated with acute, subacute and perhaps even chronic changes in brain structure 
and function (25). The persistence of post-TBI symptoms is associated with the presence and duration of these imaging abnormalities $(26,27)$. When symptoms are present, PTH is often just one symptom of many that the individual with mTBI experiences. In addition to PTH, mTBI is often associated with alterations in mood, cognition, sleep patterns, and non-headache pain (28). The presence of brain imaging abnormalities due to the mTBI itself and the common scenario of PTH being one of a constellation of symptoms, makes it challenging to isolate the brain imaging findings associated with PTH from those associated with the mTBI or other symptoms. Research designs that include cohorts who have mTBI without active symptoms and mTBI with symptoms not including headache, as well as longitudinal designs that include imaging before and after resolution of symptoms could help identify imaging findings specifically associated with PTH.

Additional studies are necessary to determine if imaging findings associated with PTH are shared by primary headaches, by other secondary headaches, or by non-headache pain types, or if there are imaging findings that are specific for PTH. This determination would lend insights into the pathophysiology of PTH and could contribute to the objective classification of PTH via models that include brain imaging data.

It is not yet known if the mechanism of brain injury leading to PTH impacts research imaging findings. A study by Obermann and colleagues measured regional gray matter using voxel based morphometry in 32 patients who had $\mathrm{PTH}$ attributed to whiplash (12). In this longitudinal study, patients were imaged within 14 days of injury and again after 3 months; those with headaches that lasted longer than 3 months $(n=12)$ were imaged again at 1 year post-injury. When imaged within the first 14 days post-injury, there were no significant regional differences in gray matter density between those with PTH and controls. For those who went on to have PPTH, there were significant decreases in gray matter density in the anterior cingulate cortex and dorsolateral prefrontal cortex when comparing the 14 days to 3 months post-injury scans. The 3-month changes in gray matter density resolved by 1 year, coinciding with headache resolution in all but one patient. The temporal correlation between the structural findings with the presence, persistence and resolution of PTH strongly suggest that the imaging findings are directly related to PTH. Furthermore, although no direct comparisons have been made between PTH attributed to whiplash with PTH attributed to TBI, the anterior cingulate cortex and dorsolateral

\section{REFERENCES}

1. Headache Classification Committee of the International Headache Society (IHS). The International Classification of Headache Disorders, 3rd edition. Cephalalgia. (2018) 38:1-211. doi: 10.1177/0333102417738202

2. Lucas S, Hoffman JM, Bell KR, Dikmen S. A prospective study of prevalence and characterization of headache following mild traumatic brain injury. Cephalalgia. (2014) 34:93-102. doi: 10.1177/0333102413499645

3. Dwyer B. Posttraumatic headache. Semin Neurol. (2018) 38:619-26. doi: $10.1055 / \mathrm{s}-0038-1673692$ prefrontal cortex are regions that have been commonly identified as participating in headache physiology as well as non-headache pain physiology $(29,30)$.

The ability to predict which individuals with acute PTH will have persistence of PTH would be a major advance for the clinical management of individuals with acute PTH and for optimizing enrollment of individuals into clinical trials of PTH therapeutics. Although much work needs to be done to understand the optimal treatments for acute and persistent PTH, presumably, the early identification of the patient who is likely to have persistence of PTH would allow for earlier and more aggressive management of acute PTH with pharmacologic and nonpharmacologic therapies. Earlier therapeutic intervention might be more effective than treatment that is started after PTH has already become persistent and might even prevent the persistence of PTH. Furthermore, to reduce unnecessary risks and to limit required patient sample sizes, it would be optimal if clinical trials of preventive therapies for acute PTH could enroll patients who are at high risk of having PTH persistence, excluding those likely to have natural PTH resolution. A prognostic biomarker for PPTH could assist with appropriate enrollment into such clinical trials.

\section{CONCLUSIONS}

Research neuroimaging is starting to provide insights into the pathophysiology of acute and persistent PTH. Further work is needed to determine if there are imaging findings that are specific to $\mathrm{PTH}$, as opposed to being attributable to the underlying trauma or post-injury symptoms that accompany PTH. Determining the specificity of imaging findings for PTH will deepen our understanding of PTH pathophysiology and might contribute to the ability to diagnostically classify new onset PTH from worsening of a pre-injury primary headache. Furthermore, imaging might contribute to models that predict which individuals with acute PTH are likely to develop PPTH, a prediction that would be useful for determining how aggressive to manage those with acute PTH and for determining a cohort of individuals who would be most appropriate for clinical trials of PTH therapeutics.

\section{AUTHOR CONTRIBUTIONS}

The author confirms being the sole contributor of this work and has approved it for publication. 
7. Alhilali LM, Delic J, Fakhran S. Differences in callosal and forniceal diffusion between patients with and without postconcussive migraine. Am J Neuroradiol. (2017) 38:691-5. doi: 10.3174/ajnr.A5073

8. Delic J, Alhilali LM, Hughes MA, Gumus S, Fakhran S. White matter injuries in mild traumatic brain injury and posttraumatic migraines: diffusion entropy analysis. Radiology. (2016) 279:859-66. doi: 10.1148/radiol.20151 51388

9. Ghodadra A, Alhilali L, Fakhran S. Principal component analysis of diffusion tensor images to determine white matter injury patterns underlying postconcussive headache. Am J Neuroradiol. (2016) 37:274-8. doi: 10.3174/ajnr.A4505

10. Yuan K, Qin W, Liu P, Zhao L, Yu D, Zhao L, et al. Reduced fractional anisotropy of corpus callosum modulates inter-hemispheric resting state functional connectivity in migraine patients without aura. PLOS ONE. (2012) 7:e45476. doi: 10.1371/journal.pone.0045476

11. Moskowitz MA, Nozaki K, Kraig RP. Neocortical spreading depression provokes the expression of $\mathrm{c}$-fos protein-like immunoreactivity within trigeminal nucleus caudalis via trigeminovascular mechanisms. J Neurosci. (1993) 13:1167-77. doi: 10.1523/JNEUROSCI.13-03-01167.1993

12. Obermann M, Nebel K, Schumann C, Holle D, Gizewski ER, Maschke M, et al. Gray matter changes related to chronic posttraumatic headache. Neurology. (2009) 73:978-83. doi: 10.1212/WNL.0b013e3181b8791a

13. Sarmento E, Moreira P, Brito C, Souza J, Jevoux C, Bigal M. Proton spectroscopy in patients with post-traumatic headache attributed to mild head injury. Headache. (2009) 49:1345-52. doi: 10.1111/j.1526-4610.2009. 01494.x

14. Niu X, Bai L, Sun Y, Wang S, Cao J, Sun C, et al. Disruption of periaqueductal grey-default mode network functional connectivity predicts persistent post-traumatic headache in mild traumatic brain injury. $J$ Neurol Neurosurg Psychiatry. (2019) 90:326-32. doi: 10.1136/jnnp-2018318886

15. Basbaum AI, Fields HL. Endogenous pain control systems: brainstem spinal pathways and endorphin circuitry. Annu Rev Neurosci. (1984) 7:309-38. doi: 10.1146/annurev.ne.07.030184.001521

16. Jang SH, Park SM, Kwon HG. Relation between injury of the periaqueductal gray and central pain in patients with mild traumatic brain injury: observational study. Medicine. (2016) 95:e4017. doi: 10.1097/MD.0000000000004017

17. Chen Z, Chen X, Liu M, Liu S, Ma L, Yu S. Disrupted functional connectivity of periaqueductal gray subregions in episodic migraine. J Headache Pain. (2017) 18:36. doi: 10.1186/s10194-0170747-9

18. Chong CD, Berisha V, Chiang CC, Ross K, Schwedt TJ. Less cortical thickness in patients with persistent post-traumatic headache compared with healthy controls: an MRI study. Headache. (2018) 58:53-61. doi: 10.1111/head. 13223

19. Chong CD, Peplinski J, Berisha V, Ross K, Schwedt TJ. Differences in fibertract profiles between patients with migraine and those with persistent post-traumatic headache. Cephalalgia. (2019) 2019:333102418815650. doi: $10.1177 / 0333102418815650$
20. Gilkey SJ, Ramadan NM, Aurora TK, Welch KM. Cerebral blood flow in chronic posttraumatic headache. Headache. (1997) 37:583-7. doi: 10.1046/j.1526-4610.1997.3709583.x

21. Schwedt TJ, Chong CD, Peplinski J, Ross K, Berisha V. Persistent post-traumatic headache vs. migraine: an MRI study demonstrating differences in brain structure. J Headache Pain. (2017) 18:87. doi: 10.1186/s10194-017-0796-0

22. Dumkrieger G, Chong CD, Ross K, Berisha V, Schwedt TJ. Static and dynamic functional connectivity differences between migraine and persistent post-traumatic headache: a resting-state magnetic resonance imaging study. Cephalalgia. (2019) 2019:333102419847728. doi: 10.1177/0333102419847728

23. Albaugh MD, Orr C, Nickerson JP, Zweber C, Slauterbeck JR, Hipko S, et al. Postconcussion symptoms are associated with cerebral cortical thickness in healthy collegiate and preparatory school ice hockey players. J Pediatr. (2015) 166:394-400 e1. doi: 10.1016/j.jpeds.2014.10.016

24. D'Souza MM, Trivedi R, Singh K, Grover H, Choudhury A, Kaur P, et al. Traumatic brain injury and the post-concussion syndrome: a diffusion tensor tractography study. Indian J Radiol Imaging. (2015) 25:404-14. doi: 10.4103/0971-3026.169445

25. Chong CD, Schwedt TJ. Research imaging of brain structure and function after concussion. Headache. (2018) 58:827-35. doi: 10.1111/head.13269

26. Messe A, Caplain S, Pelegrini-Issac M, Blancho S, Montreuil M, Lévy $\mathrm{R}$, et al. Structural integrity and postconcussion syndrome in mild traumatic brain injury patients. Brain Imaging Behav. (2012) 6:283-92. doi: 10.1007/s11682-012-9159-2

27. Hartikainen KM, Waljas M, Isoviita T, Dastidar P, Liimatainen S, Solbakk AK, et al. Persistent symptoms in mild to moderate traumatic brain injury associated with executive dysfunction. J Clin Exp Neuropsychol. (2010) 32:767-74. doi: 10.1080/13803390903521000

28. Ruff RL, Riechers RG II, Wang XF, Piero T, Ruff SS. A case-control study examining whether neurological deficits and PTSD in combat veterans are related to episodes of mild TBI. BMJ Open. (2012) 2:e000312. doi: 10.1136/bmjopen-2011-000312

29. Becerra L, Veggeberg R, Prescot A, Jensen JE, Renshaw P, Scrivani S, et al. A 'complex' of brain metabolites distinguish altered chemistry in the cingulate cortex of episodic migraine patients. Neuroimage Clin. (2016) 11:588-94. doi: 10.1016/j.nicl.2016.03.020

30. Vogt BA. Pain and emotion interactions in subregions of the cingulate gyrus. Nat Rev Neurosci. (2005) 6:533-44. doi: 10.1038/nrn1704

Conflict of Interest Statement: The author declares that the research was conducted in the absence of any commercial or financial relationships that could be construed as a potential conflict of interest.

Copyright () 2019 Schwedt. This is an open-access article distributed under the terms of the Creative Commons Attribution License (CC BY). The use, distribution or reproduction in other forums is permitted, provided the original author(s) and the copyright owner(s) are credited and that the original publication in this journal is cited, in accordance with accepted academic practice. No use, distribution or reproduction is permitted which does not comply with these terms. 